\title{
A real-time PCR assay for rapid identification of inducible and acquired clarithromycin resistance in Mycobacterium abscessus
}

\author{
Meenu Kaushal Sharma ${ }^{1,2^{*}}$ (D, Yanni La ${ }^{3}$, Debra Janella ${ }^{1}$ and Hafid Soualhine $e^{1,2}$
}

\begin{abstract}
Background: Mycobacterium abscessus is a rapidly growing mycobacteria involved in severe infections of the lung, skin, or soft tissue. Macrolides such as clarithromycin are the recommended first line drugs for treatment of $M$. abscessus infections. However, M. abscessus has dual mechanisms of resistance to macrolides, making treatment by macrolides difficult. A functional erm(41) gene confers for inducible resistance while acquired mutations on the $23 \mathrm{~S}$ rRNA rrl gene confer for constitutive resistance.

Methods: We have developed a real-time PCR assay to detect both inducible and acquired resistance to clarithromycin, and compared the results to traditional erm(41) and rrl sequencing and phenotypic susceptibility testing using Sensititre ${ }^{\mathrm{TM}}$ plates.

Results: Of the total 126 M. abscessus isolates tested, truncated erm(41) was found in 23/126 (18.3\%) of the samples, 27/126 (21.4\%) had a T28C mutation in erm(41), and 2/126 (1.6\%) had an acquired A2058C mutation in rrl. The phenotypic results correlated with the expected sequencing results in 121/126 samples (96\%). Phenotypic testing compared to real-time PCR resolved 2 of these discrepancies by showing the existence of both erm(41) alleles in the isolates that sequencing missed. One culture was found to be mixed with two M. abscessus subsp. as per hsp65 sequencing and 2 isolates had discordance between molecular and phenotypic results. It was presumed that 3 isolates showed discrepancy between sequencing and real-time PCR, but one culture was mixed and other 2 detected both alleles by real-time PCR leading to $100 \%$ concordance when compared to sequencing.
\end{abstract}

Conclusion: In conclusion, real-time PCR is more accurate for detection of both acquired and induced clarithromycin resistance, specifically when mixed genic profiles are present in a sample.

Keywords: Mycobacterium abscessus, Real-time PCR, erm(41), Clarithromycin

\footnotetext{
* Correspondence: Meenu.Sharma@canada.ca

'National Reference Centre for Mycobacteriology, National Microbiology Laboratory, Public Health Agency of Canada, 1015 Arlington St, R3E 3R2 Winnipeg, Canada

${ }^{2}$ Department of Medical Microbiology, University of Manitoba, Winnipeg,

Canada

Full list of author information is available at the end of the article
}

(c) The Author(s). 2020 Open Access This article is licensed under a Creative Commons Attribution 4.0 International License, which permits use, sharing, adaptation, distribution and reproduction in any medium or format, as long as you give appropriate credit to the original author(s) and the source, provide a link to the Creative Commons licence, and indicate if changes were made. The images or other third party material in this article are included in the article's Creative Commons licence, unless indicated otherwise in a credit line to the material. If material is not included in the article's Creative Commons licence and your intended use is not permitted by statutory regulation or exceeds the permitted use, you will need to obtain permission directly from the copyright holder. To view a copy of this licence, visit http://creativecommons.org/licenses/by/4.0/. The Creative Commons Public Domain Dedication waiver (http://creativecommons.org/publicdomain/zero/1.0/) applies to the data made available in this article, unless otherwise stated in a credit line to the data. 


\section{Background}

As per former and accepted taxonomy, the rapidly growing Mycobacterium abscessus is subdivided to 3 subspecies $M$. abscessus subsp. abscessus, M. abscessus subsp. bolletii, and $M$. abscessus subsp. massiliense which are involved in infections of the skin, lung, as well as infections associated with medical procedures [1, 2]. These infections are commonly treated with macrolides (clarithromycin, azithromycin and erythromycin) but resistance to macrolides makes treatment increasingly difficult $[3,4]$. M. abscessus infections management may require multi-drug therapy along with intravenous treatment for several months. Intravenous agents such as amikacin are commonly associated with side effects in patients [5]. Identifying resistance earlier can determine the best suited treatment. According to the Clinical Laboratory Standard Institute (CLSI), drug susceptibility testing for clarithromycin require an incubation time of up to 14 days to assess inducible resistance [6].

Natural and acquired resistance to clarithromycin are due to the erythromycin ribosomal methyl transferase, erm(41), and the gene encoding a 23S peptidyl transferase in the large $23 \mathrm{~S}$ ribosomal subunit, $r r l$, respectively [7-9]. Inducible resistance occurs naturally with a functional erm(41) gene [7]. However, a common trait of $M$. abscessus subsp. massiliense isolates is that they have a 274-bp deletion in erm(41), making it non-functional, hence susceptible to macrolides [7]. Thus, M. abscessus subspecies identification and determination of macrolide susceptibility are useful in the planning of the appropriate treatment. Another mutation, the $\mathrm{T}$ to $\mathrm{C}$ mutation at position 28 on $\operatorname{erm}(41)$ also leads to a non-functional erythromycin ribosomal methyl transferase [7]. When inducible resistance occurs, drug susceptibility testing will show susceptibility in vitro at day 3 but will later develop resistance up to 14 days post-incubation. Clinically acquired resistance to a macrolide occurs from a spontaneous mutation at positions 2058 and 2059 on the $\mathrm{rrl}$ gene of 23S, which causes an alteration to the drug binding pocket of the protein $[8,9]$. Constitutive resistance will result in a high minimum inhibitory concentration (MIC) at day 3 of susceptibility testing. As reported by Vester et al. (2001), acquired mutations at positions 2057 and 2611 on $\mathrm{rrl}$ can also result in low level resistance due to being in close proximity to the action centre [10]. These mutations are outside the focal point of macrolide interaction but can still disrupt the structure of the drug binding pocket, reducing its ability to inhibit the ribosome [10].

Sequencing of $16 \mathrm{~S}$ and $h s p 65$ (heat shock protein 65) genes was also used to identify the different subspecies of $M$. abscessus. Sequencing of erm(41) and $r r l$ was done and probes for real-time PCR were designed to look for possible mutations that would cause resistance to clarithromycin. A real-time PCR assay was designed to run under a single condition which would greatly decrease the turnaround time of 14 days using phenotypic testing, to just a few hours to predict resistance to clarithromycin. Real-time PCR assay was also compared to the sequencing method currently used in our laboratory.

\section{Methods}

\section{Identification of M. abscessus subspecies}

$M$. abscessus isolates identification was performed using $16 \mathrm{~S}$ gene sequencing and the subsp. identification was done using sequencing of the hsp 65 gene. PCR amplification was done in a $50 \mu \mathrm{L}$ reaction volume consisting of $47.5 \mu \mathrm{L}$ premix $(1 \mu \mathrm{M}$ forward primer, $1 \mu \mathrm{M}$ reverse primer), $0.25 \mu \mathrm{L}$ of Qiagen Taq DNA polymerase (Qiagen, Hilden, Germany), and $2.5 \mu \mathrm{L}$ of template DNA (Table 1). Amplification was done using the Applied Biosystems Veriti $^{\odot}$ 96-Well Thermal Cycler (Thermo Fisher Scientific, Applied Biosystems, Grand Island, NY). Thermocycler conditions for $h s p 65$ were $95^{\circ} \mathrm{C}$ for $5 \mathrm{~min}$, 45 cycles of $95^{\circ} \mathrm{C}$ for $1 \mathrm{~min}, 60^{\circ} \mathrm{C}$ for $1 \mathrm{~min}$, and $72{ }^{\circ} \mathrm{C}$ for $1 \mathrm{~min}$, followed by $72{ }^{\circ} \mathrm{C}$ for $5 \mathrm{~min}$. The PCR products were purified using PCRClean DX magnetic beads (Aline Biosciences, Woburn, MA). Sequencing was done using Applied Biosystems 3730xl DNA Analyzer (Thermo Fisher Scientific, Applied Biosystems, Grand Island, NY). Sequences were assembled using Lasergene SeqMan Pro (DNAStar, Inc., Madison, WI) and sequence comparison was done using BioNumerics 7.6.2 software. Reference strains Mycobacterium abscessus subsp. abscessus ATCC $19977^{\mathrm{T}}$, Mycobacterium abscessus subsp. bolletii CCUG $50184^{\mathrm{T}}$, and Mycobacterium abscessus subsp. massiliense CCUG $48898^{\mathrm{T}}$ were used.

\section{Antimicrobial susceptibility testing}

Phenotypic susceptibility testing for clarithromycin was done using Sensititre ${ }^{\text {тм }}$ RAPMYCO AST plates (Trek Diagnostics, Thermo Fisher Scientific, Oakwood Village, $\mathrm{OH}$ ) according to the manufacturer instructions. Plates were examined for the drug MICs on day 3 (and day 5 if growth in positive control well was not adequate) of incubation then further incubation at $30^{\circ} \mathrm{C}$ was done if day 3 or 5 results showed sensitivity. Plates were examined for inducible resistance on day 7, 10 and 14 of incubation.

\section{Sequencing of erm(41) and $r r l$ genes}

PCR amplification was done in a total reaction volume of $50 \mu \mathrm{L}$ which consisted of $25 \mu \mathrm{L}$ Amplitaq Gold 360 Mastermix (Thermo Fisher Scientific, Applied Biosystems, Foster City, CA), $22.5 \mu \mathrm{L}$ forward and reverse primers (final concentration of $1 \mu \mathrm{M}$ each), and $2.5 \mu \mathrm{L}$ of template DNA for both erm(41) and $r r l$ genes. 
Table 1 Primers used for sequencing of hsp65, erm(41) and $r r l$ of the M. abscessus

\begin{tabular}{|c|c|c|c|c|}
\hline Primer & Target Gene & Sequence & Annealing Temp $\left({ }^{\circ} \mathrm{C}\right)$ & Reference \\
\hline TB11 & hsp65 & 5'-ACCAACGATGGTGTGTCCAT-3' & 60 & [11] \\
\hline TB12 & hsp65 & 5'-CTTGTCGAAGGCCATACCCT-3' & 60 & [11] \\
\hline ermF & $\operatorname{erm}(41)$ & 5'-GACCGGGGCCTTCTTCGTGAT-3' & 60 & [12] \\
\hline ermR1 & $\operatorname{erm}(41)$ & 5'-GACTTCCCCGCACCGATTCC-3' & 60 & [12] \\
\hline erm41-2 & $\operatorname{erm}(41)$ & 5'-GGATTCCGGCGTCAAGAGACTC-3' & 60 & [9] \\
\hline erm41-3 & $\operatorname{erm}(41)$ & 5'-CGAGCCCGCCCTACCAAGTCAC-3' & 60 & [7] \\
\hline erm41-4 & $\operatorname{erm}(41)$ & 5'-CCGGCCCGTAGCGTCCAATG-3' & 60 & [13] \\
\hline erm41-5 & $\operatorname{erm}(41)$ & 5'-ACTCCCCTGAGCGA ACAC-3' & 60 & [7] \\
\hline $19 F$ & $r r l$ & 5'-GTAGCGAAATTCCTTGTCGG-3' & 55 & [14] \\
\hline $21 R$ & $r r l$ & 5'-TTCCCGCTTAGATGCTTTCAG-3' & 55 & [14] \\
\hline SP1 & $r r l$ & 5'-CCTGCACGAATGGCGTAACG-3' & 55 & [15] \\
\hline SP2mod & $r r l$ & 5'-CACCAGAGGTTCGTCCGTC-3' & 55 & {$[15]$} \\
\hline
\end{tabular}

Primers ermF and ermR1 were used to amplify the erm(41) gene while primers $19 \mathrm{~F}$ and $21 \mathrm{R}$ amplified the $r r l$ gene (Table 1). Thermocycler conditions for amplifying erm(41) were $95^{\circ} \mathrm{C}$ for $7.5 \mathrm{~min}$ for the initial denaturation, followed by 35 cycles of $95^{\circ} \mathrm{C}$ for $30 \mathrm{~s}, 60^{\circ} \mathrm{C}$ for $30 \mathrm{~s}$, and $72^{\circ} \mathrm{C}$ for $30 \mathrm{~s}$ followed by a final extension of $72{ }^{\circ} \mathrm{C}$ for $10 \mathrm{~min}$. Conditions were the same for $\mathrm{rrl}$ except with an annealing temperature of $55^{\circ} \mathrm{C}$ instead of $60^{\circ} \mathrm{C}$. The PCR products were purified using PCRClean DX magnetic beads. Sequencing primers are shown in Table 1. Sequencing and analysis were done as described above for $h s p 65$ gene.

\section{Real-time PCR assay}

A 96-well plate assay was designed based on a modified protocol from Shamira Shallom and colleagues (2015)
[11]. The probe, Absc-chel 16S, was used for identification, erm(41)_probe1 was used to detect full-length erm(41), SNPs on position 28 of erm(41) were detected using probes erm(41)T28 and erm(41)C28, SNPs on position 2058 of $r r l$ were detected using probes 23S_A2058 and 23S_C2058 (Table 2). Probes for $r r l$, 23S_A2058 and 23S_C2058, initially consisted of LNA (locked nucleic acids) from the Shallom protocol, however, the $r r l$ probes used in this experiment were modified. This change would see a lower melting temperature for these probes and therefore, the $5^{\prime}$ end of these probes were extended by 7 base pairs using Mabs5 23S rRNA (GenBank accession number EU980535.1) to ensure the same melting temperature of around $60^{\circ} \mathrm{C}$. Probes were made to a working concentration of $2.5 \mu \mathrm{M}$ in a mix with their corresponding primers (Table 2) which were diluted to

Table 2 Probes and primers used in the real-time assay for M. abscessus

\begin{tabular}{|c|c|c|c|}
\hline Target & & Sequence & Reference \\
\hline \multirow[t]{3}{*}{$16 S$} & Absc-chel 16S & 5'-6FAM-ACC ACA CAC TTC A-MGB-NFQ-3' & {$[16]$} \\
\hline & F Primer16S & 5'-ATAAGCCTGGGAAACTGGGTCTA-3' & \\
\hline & R Primer16S & 5'-CCACACCGCAAAAGCTTT-3' & \\
\hline \multirow[t]{3}{*}{ Full length erm(41) } & erm(41)_probe1 & 5'-6FAM-TGC TAG CCG TCG AGC TGC ATC C-QSY-3' & {$[17]$} \\
\hline & F PrimerTR & 5'-TCAGGGGAGTTCGTTGTGGAT-3' & \\
\hline & R PrimerTR & 5'-TCTTCCTCGGCAAACCGT-3' & \\
\hline \multirow[t]{4}{*}{$\operatorname{erm}(41)$} & $\operatorname{erm}(41) \mathrm{T} 28$ & $5^{\prime}-\mathrm{HEX}-\mathrm{CCA}+\mathrm{G}+\mathrm{T}+\mathrm{G}$ GGG C-IABkFQ-3' & [12] \\
\hline & $\operatorname{erm}(41) C 28$ & 5'-6FAM-CCA + G + C + G GGGC-IABkFQ-3' & \\
\hline & F PrimerE28 & 5'-GAGCATGGGCATATTCATGATGG-3' & \\
\hline & R PrimerE28 & 5'-TGAGCGAACACCGGATTCG-3' & \\
\hline \multirow[t]{4}{*}{$r r l$} & 23S_A2058 & 5'-6FAM-CGGCAGGACGAAAAGACCC-BHQ1-3' & {$[12]$} \\
\hline & 23S_C2058 & 5'-6FAM-CGGCAGGACGACAAGACCC-BHQ1-3' & \\
\hline & F PrimerR2058 & 5'-GCGAAATTGCACTACGAGTAAAG-3' & \\
\hline & R PrimerR2058 & 5'-CCTATCCTACACAAACCGAACC-3' & \\
\hline
\end{tabular}


$10 \mu \mathrm{M}$. Reactions were done in a total reaction volume of $25 \mu \mathrm{L}$ consisting of $12.5 \mu \mathrm{L}$ TaqMan ${ }^{\odot}$ Fast Advanced Master Mix (Thermo Fisher Scientific, Life Technologies, Austin, TX.), probes final concentration of $250 \mathrm{nM}$ and forward and reverse primers at final concentration of $1 \mu \mathrm{M}$ each, and $3.75 \mu \mathrm{L}$ of template DNA. Each sample was run in duplicates with positive control for all wild type and mutants i.e., 16S, full length erm(41), T28T in erm(41), T28C in erm(41), A2058A in rrl, and A2058C in $r r l$ and negative control (no sample DNA). Real-time PCR was done using the Applied Biosystems QuantStudio 3 Real-Time PCR System (Thermo Fisher Scientific, Applied Biosystems, Grand Island, NY). Thermocycler conditions included an initial denaturation of $95^{\circ} \mathrm{C}$ for $3 \mathrm{~min}, 40$ cycles of $95^{\circ} \mathrm{C}$ for $30 \mathrm{~s}, 60^{\circ} \mathrm{C}$ for $30 \mathrm{~s}$, and $72{ }^{\circ} \mathrm{C}$ for $30 \mathrm{~s}$.

\section{Results}

The hsp65 sequences were compared using Bionumerics version 7.6.2 (Applied Math, Belgium). Sequential differences in $h s p 65$ were used to distinguish the three members of $M$. abscessus as described in Kazue Nakanaga et al. (2014) [18]. It was found that in our 126 samples, 94 were identified as $M$. abscessus subsp. abscessus, $7 \mathrm{M}$. abscessus subsp. bolletii, 23 M. abscessus subsp. massiliense, and 2 showed the coexistence of two mixed DNA populations belonging to $M$. abscessus subspecies.

Clarithromycin susceptibility testing using microbroth dilution assay found that of the 126 isolates, 3 were resistant on day 3 ( 2 M. abscessus subsp. abscessus, $1 \mathrm{M}$. abscessus subsp. massiliense), 76 were inducibly resistant (70 M. abscessus subsp. abscessus, 5M. abscessus subsp. bolletii, $1 \mathrm{M}$. abscessus subsp. massiliense). One isolate with intermediate result (M. abscessus subsp. abscessus) was repeated with an updated phenotypic result showing susceptible result giving a total of 47 susceptible results (22 M. abscessus subsp. abscessus, 2 M. abscessus subsp. bolletii, $21 \mathrm{M}$. abscessus subsp. massiliense, 2 mixed by hsp65 sequencing) (Table 3). Inducible resistance is characterized by an MIC of 8 or greater observed on day

Table 3 Clarithromycin Minimum Inhibitory Concentration (MIC) interpretations for M. abscessus isolates

\begin{tabular}{lllll}
\hline & \multicolumn{3}{l}{ MIC Interpretation } & \\
\cline { 2 - 4 } hsp65 Identification & S & IR & $\mathbf{R}$ & Total \\
\hline M. abscessus subsp. abscessus & $22^{\text {a }}$ & 70 & 2 & $94^{\text {a }}$ \\
M. abscessus subsp. bolletii & 2 & 5 & - & 7 \\
M. abscessus subsp. massiliense & 21 & 1 & 1 & 23 \\
M. abscessus subspecies mixed & 2 & - & - & 2 \\
Total & $\mathbf{4 7}$ & $\mathbf{7 6}$ & $\mathbf{3}$ & $\mathbf{1 2 6}$
\end{tabular}

S, Sensitive (MIC $\leq 2$ ); IR, Inducible Resistance (MIC $\geq 8$ on day 7 or later of susceptibility testing); R, Resistant (MIC $\geq 8$ on day 3 of susceptibility testing); ${ }^{a} 2$ mixed cultures of $M$. abscessus subsp. abscessus, each found to have both wild type T28 and mutation C28 in erm(41)
7 and up to day 14 after an initial result of susceptibility at day 3 [19].

\section{Sequencing of erm(41) and $r r l$ for mutations}

Sequencing found that 23 samples had the 274-bp deletion on $\operatorname{erm}(41)$. Twenty-two of which were found to be in the subspecies $M$. abscessus subsp. massiliense. One was in a mixed population containing Mycobacterium abscessus subsp. massiliense (Table 4). Twenty-seven strains had the T28C mutation in erm(41) (24 M. abscessus subsp. abscessus, $2 \mathrm{M}$. abscessus subsp. bolletii, and 1 mixed) and 2 strains harboured a A2058C mutations on rrl (1M. abscessus subsp. abscessus and $1 M$. abscessus subsp. massiliense) (Table 4). Five strains (M. abscessus subsp. abscessus) had discrepant results which had phenotypic results that did not match with the mutation found with sequencing, which have been explained in the discussion section.

\section{Real-time PCR assay}

Our real-time assay was optimized by modifying Shallom 2015 protocol. Our method ran all of the targeted mutations of a sample on a single plate and under the same PCR conditions which include detection of full-length erm(41). Shallom et al. used a SYBR green quantitative method separate from their probe based one for detecting full-length erm(41). Additionally, we used a probe for species confirmation in our assay, making our assay far more informative in a single run. The probes for the $r r l$ targets originally consisted of LNA bases, but due to availability, ours consist of regular DNA bases. The probes' length was then changed from 12 to 19 base pairs to ensure a matching melting temperature with other reactions on the plate. This has impact on the real-time PCR conditions as we intend to multiplex this assay at a later date. Black Hole quencher probe tags were also used in place of Iowa Black fluorescent tags for the modified $r r l$ probes. These modified probes were locally produced in our facility and therefore more cost effective and allowed the assessment of different probe and conditions. Results of the real-time assay showed amplification for all our targets using the aforementioned probes under the same cycling conditions. Successful detection in our $r r l$ targets informs us that LNA bases in probes is not necessary to perform this assay. Crossing threshold (CT) values were found to range between 16 and 24 cycles for different reactions (Fig. 1). Samples were run in duplicates and CT values of duplicates showed minimal variability of one CT with orange color having slightly more variability between duplicates.

When real-time PCR assay was compared to sequencing, one discrepant sample had a truncated erm(41) gene with the $\mathrm{T}$ allele with sequencing, but real-time PCR showed detection of a full-length gene and a $\mathrm{C}$ 
Table 4 Comparisons of mutations found in erm(41) and rrl for each subspecies of the $M$. abscessus using sequencing and real-time PCR

\begin{tabular}{|c|c|c|c|c|c|c|c|c|}
\hline \multicolumn{3}{|c|}{ Phenotypic Result } & \multicolumn{3}{|l|}{ Sequencing Results } & \multicolumn{3}{|l|}{ Real-time PCR Results } \\
\hline Subspecies & \# of Samples & & $\begin{array}{l}\text { Full-length/truncated } \\
\text { erm } 41\end{array}$ & $\begin{array}{l}\text { erm41 } \\
\text { position } 28\end{array}$ & $\begin{array}{l}\text { rrl position } \\
2058\end{array}$ & $\begin{array}{l}\text { Full-length/truncated } \\
\text { erm41 }\end{array}$ & $\begin{array}{l}\text { erm41 } \\
\text { position } 28\end{array}$ & $\begin{array}{l}\text { rrl position } \\
2058\end{array}$ \\
\hline \multicolumn{9}{|c|}{ M. abscessus subsp. abscessus } \\
\hline & 67 & $\mathbb{R}$ & Full & $\mathrm{T}$ & A & Full & T & A \\
\hline & 1 & $R^{a}$ & Full & $\mathrm{T}$ & A & Full & T & A \\
\hline & 1 & $S^{a}$ & Full & $\mathrm{T}$ & A & Full & T & A \\
\hline & 1 & $\mathrm{R}$ & Full & $\mathrm{T}$ & C & Full & T & C \\
\hline & 21 & S & Full & C & A & Full & C & A \\
\hline & 2 & $\mathbb{R}$ & Full & $C$ & A & Full & $T+C$ & A \\
\hline & 1 & $\mathbb{R}^{\mathrm{a}}$ & Full & C & A & Full & C & A \\
\hline \multicolumn{9}{|c|}{ M. abscessus subsp. bolletii } \\
\hline & 5 & $\mathbb{R}$ & Full & T & A & Full & T & A \\
\hline & 2 & S & Full & C & A & Full & C & A \\
\hline \multicolumn{9}{|c|}{ M. abscessus subsp. massiliense } \\
\hline & 21 & S & Truncated & $\mathrm{T}$ & A & Truncated & T & A \\
\hline & 1 & $\mathrm{R}$ & Truncated & $\mathrm{T}$ & C & Truncated & T & C \\
\hline & 1 & $\mathbb{R}$ & Full & $\mathrm{T}$ & A & Full & T & A \\
\hline \multicolumn{9}{|c|}{${ }^{\mathrm{b}}$ M. abscessus } \\
\hline & 1 & S & Full & C & A & Full & C & A \\
\hline & 1 & S & Truncated & $\mathrm{T}$ & A & Full & $C$ & A \\
\hline
\end{tabular}

${ }^{a}$ Discrepant phenotypic result to what was expected with real-time results

${ }^{\mathrm{b}} M$. abscessus subspecies with two hsp65 gene sequences

allele. Both methods found no mutations on position 2058 of the $r r l$ gene. This strain was found to be mixed with $M$. abscessus subsp. abscessus and $M$. abscessus subsp. massiliense as per hsp65 sequencing data. Two isolates of M. abscessus subsp. abscessus had discordant sequencing and phenotypic results, with both $\mathrm{T}$ and $\mathrm{C}$ alleles of erm(41) amplified with real-time PCR and only T28C mutation with sequencing. Hence, only sequencing showed discrepancy to the phenotypic results but real-time PCR did not as the latter detected both alleles. Real-time PCR results resolved all these three discrepancies.

\section{Discussion}

Clarithromycin is an important antibiotic against members of the M. abscessus [3]. Resistance to macrolide can be predominantly correlated with two genes, erm(41) and $r r l$ in $M$. abscessus. The full erm(41) gene that is without the $274 \mathrm{bp}$ deletion and $\mathrm{T}$ at position 28 will result in a functional erythromycin ribosomal methyl transferase and hence show inducible resistance [7]. Extended periods of incubation in the antibiotic will induce resistance in $M$. abscessus isolates that have an inducible resistance genic profile. An acquired point mutation on positions 2058 and/or 2059 of $r r l$ will result in high level resistance against clarithromycin by altering the drug binding domain of the $23 \mathrm{~S}$ rRNA $[8,9]$. These are characteristically shown by resistance (MIC $\geq 8$ ) on day 3 of antimicrobial susceptibility testing and hence indicate constitutive resistance.

Phenotypic susceptibility testing was done by broth microdilution and samples were incubated for up to 14 days [6]. Initial testing found that 79 (62.7\%) samples were resistant of which 76 (60.3\%) showed inducible resistance. Forty-six were initially shown to be susceptible at day 3; however, one sample with an initial intermediate result was repeated and showed to be sensitive for a total of $47(37.3 \%)$ sensitive samples. For this intermediate sample, molecular methods found it to have a T28C mutation so a susceptible result was expected and overgrowth in the initial phenotypic testing may be the cause for the intermediate reading. In the 2 strains that were determined to be mixed by $h s p 65$ sequencing, both were susceptible to clarithromycin.

Sequencing of erm(41) and $r r l$ identified 27 strains (21.4\%) with a T28C mutation, 23 (18.3\%) had a truncated erm(41) gene and $2(1.6 \%)$ with the A2058C mutation on rrl. The 274-bp deletion on erm(41) was identified in only $M$. abscessus subsp. massiliense and 1 mixed $M$. abscessus sample containing M. abscessus 


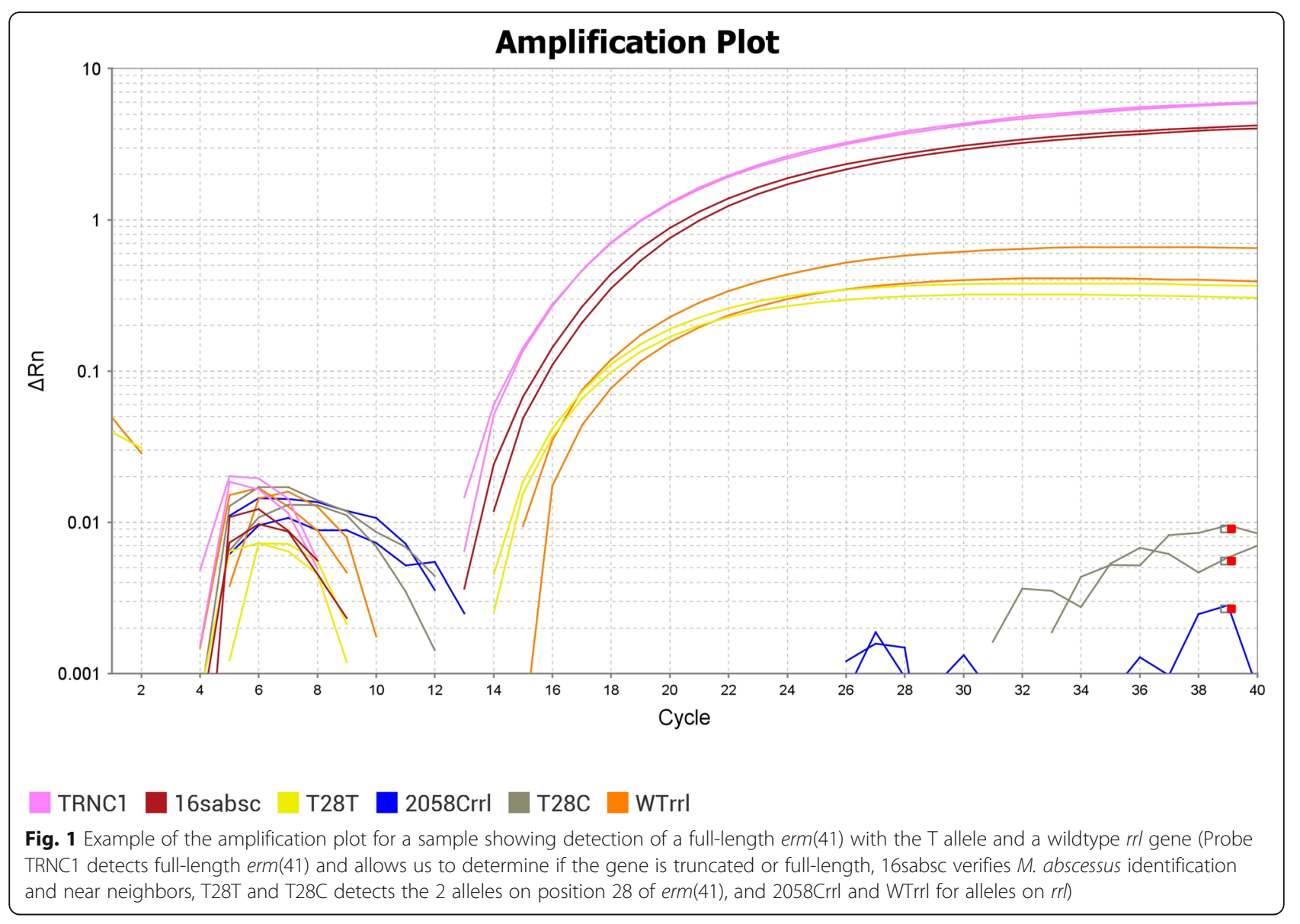

subsp. massiliense. This deletion is characteristic of $M$. abscessus subsp. massiliense as reported by Hee-youn Kim et al. (2010) which is why clarithromycin treatment is found to be more effective against infections with $M$. abscessus subsp. massiliense than the other subspecies that contain the functional full-length gene [20]. One sample in our study that was identified as $M$. abscessus subsp. massiliense was found to possess a full-length erm(41) gene by both sequencing and real-time PCR. Shamira Shallom and colleagues (2013) have also identified $M$. abscessus subsp. massiliense isolates with fulllength $\mathrm{erm}(41)$ in which they suggest is probably due to horizontal transfer [21].

Sequencing results of erm(41) and $r r l$ were 96.0\% (121/126) concordant with the phenotypic results, with 5 isolates of $M$. abscessus found to have phenotypic results discordant with the molecular results. Two isolates of $M$. abscessus subsp. abscessus showed inducible resistance phenotypically, but had the T28C mutation found with sequencing for an expected result to clarithromycin susceptibility. For these two isolates, both $\mathrm{T}$ and $C$ alleles on position 28 of erm(41) were detected in the real-time PCR assay, showing that both alleles were present and that the real-time PCR method uses a probe based detection and hence was more sensitive than sequencing. Our sequencing method only detected the predominant $\mathrm{C}$ allele for this sample as in general, a sequencing method will more frequently utilize DNA template containing a predominant allele. However, realtime PCR can detect both most and less predominant alleles in low DNA concentrations. Another isolate showed an inducible resistant result phenotypically without a corresponding allele. This could not be resolved when both phenotypic and molecular assays were repeated and is likely due to another mutation outside the gene fragments used in this study. Efflux pump ability has been shown to be directly involved with clarithromycin resistance [22]. One isolate that showed a phenotypic susceptible result lacked correlation with genotypic mutations and also could not be resolved when repeated testing showed reproducibility on all assays. This is perhaps due to unknown mechanism that renders the isolate susceptible to clarithromycin. Extending the search to look for additional mutations did not show erm(41) C19T and hence evidence of susceptibility is lacking [23]. The fifth discordant result was resistant on day 3 of susceptibility testing indicating an acquired resistance to clarithromycin, but had no mutations 
found on positions 2058 of $r r l$ with sequencing. This is suspected to be caused by a point mutation elsewhere on $r r l$ that would affect the drug binding pocket. The discrepant strains that could be resolved by $h s p 65$ or real-time PCR had their clarithromycin phenotypic susceptibility testing repeated with 3 of the final results staying reproducible to the initial results. There were no additional mutations in the $r r l$ gene such as A2056G, A2057G, A2080C, G2068A, A2269G, G2281A, as the sequence matched wild type [10, 23].

A real-time assay was developed to assess resistance in $M$. abscessus isolates by targeting mutations that were found in sequencing. The assay was built upon the protocol in Shallom et al. (2015) for erm(41) and $r r l$ with primer and tag modifications that allowed us to perform amplification under a single cycling condition; and included additional probes for $16 \mathrm{~S}$ identification and full length erm(41) detection [12, 16, 17]. A probe for detecting the $16 \mathrm{~S}$ gene of $M$. abscessus was used to confirm that samples were members of the $M$. abscessus and verified that $\operatorname{erm}(41)$ is present [16]. Discrimination of full-length and truncated erm(41) was done by having a probe and the reverse primer hybridize the region of the gene that is deleted. Amplification indicates a full-length erm(41) gene [17]. Two sets of probes were used to detect the mutant and wild type alleles for each position 28 of erm(41) and position 2058 of $r r l$ [12]. With all of the included probes in our assay, identification and mutation detection can be determined for a sample after a single real-time PCR run. Compared to the 3-14 days needed for susceptibility testing, this modified real-time PCR method has significant impact in a clinical laboratory as it is much faster and helps in determining the best treatment. Samples were run in duplicates and were found to have similar $\mathrm{CT}$ and $\Delta \mathrm{Rn}$ values (Fig. 1) for each target, showing the high reproducibility of the assay and had CT values ranging from 16 to 24 for all reactions. Real-time results were compared to the sequencing data to examine adequate amplification in our assay. The real-time results confirmed the sequencing results in our samples. One sample showed a truncated erm(41) gene in sequencing, but detection of the fulllength gene was observed with real-time PCR. Upon repeating $h s p 65$ sequencing for this discrepancy, it was found to be mixed with both $M$. abscessus subsp. abscessus and M. abscessus subsp. massiliense. Hence, both test results were correct. Two samples detected both alleles of erm(41) by real-time PCR whereas sequencing only detected single $\mathrm{C}$ allele. The phenotypic results for these samples showed inducible resistance, indicating that the $\mathrm{T}$ allele was present and only real-time PCR was sensitive enough to detect it compared to sequencing. Hence, real-time PCR was superior to sequencing as it requires smaller DNA concentration and may detect both mutation profiles in a single test. Real-time PCR also has an advantage over the sequencing method done in our laboratory as it provides rapid test turnaround times, is less labour intensive with satisfactory test accuracy. The resulting output is a simple negative or positive for a real-time reaction i.e., looking for amplification vs interpreting sequencing chromatograms and hence less data screening. To summarize, even though 3 results were presumed discrepant between phenotypic and real-time PCR (97.6\%; 123/126); 1 was found to be mixed by hsp65 sequencing and 2 samples detected both T28 and C28 erm(41) alleles by real-time PCR. This meant that in the presumed discrepant isolates, $100 \%$ concordance between sequencing and real-time was seen due to the real-time results. Hence, real-time PCR demonstrated slight advantage over traditional sequencing assay.

\section{Conclusion}

In conclusion, a real-time detection assay shortened turnaround times compared to phenotypic susceptibility testing and traditional sequencing. Probes for detecting A2058G and A2059G mutations on $r r l$ were not appropriately evaluated in our study since incidence of $\mathrm{rrl} \mathrm{mu}$ tations were low. However, $r r l$ was implemented in our assay for future testing to detect possible mutations that may be responsible for constitutive resistance. Multiplexing this assay could also result in conservation of reagents and hence decreased cost while running more samples on one plate/run. Whether by use of sequencing or by real-time PCR assay, there is advantage in detecting erm(41) variants to predict sensitivity of $M$. abscessus isolates to clarithromycin, hence impacting treatment outcomes.

\section{Abbreviations}

PCR: Polymerase chain reaction; rRNA: ribosomal ribonucleic acid; DNA: deoxyribonucleic acid; LNA: locked nucleic acids; CT: Threshold cycle; $\Delta R n$ : Normalized reporter value minus baseline; MIC: minimum inhibitory concentration; CLSI: Clinical Laboratory Standard Institute

\section{Acknowledgements \\ The authors would like to thank all staff of National Reference Centre for Mycobacteriology, Winnipeg.}

\section{Authors' contributions}

MS developed the concept of the work, sought human resources, funding and approval for the work, analyzed, interpreted results, wrote the manuscript and obtained coauthor consent. YL performed the assay, analyses, interpreted results, wrote and edited the manuscript. DJ performed the assay and data acquisition. HS performed data analysis and interpretation. All authors read and approved the final manuscript.

\section{Funding}

Not applicable.

Availability of data and materials

The nucleotide datasets generated and/or analyzed during the current study are available in the NCBI under Banklt submission number 2402752 and accession numbers MW275120 - MW275245. 
Ethics approval and consent to participate

Not applicable.

\section{Consent for publication}

Not applicable.

\section{Competing interests}

None.

\section{Author details}

${ }^{1}$ National Reference Centre for Mycobacteriology, National Microbiology Laboratory, Public Health Agency of Canada, 1015 Arlington St, R3E 3R2 Winnipeg, Canada. ${ }^{2}$ Department of Medical Microbiology, University of Manitoba, Winnipeg, Canada. ${ }^{3}$ Department of Microbiology, University of Manitoba, Winnipeg, Canada.

Received: 26 June 2020 Accepted: 4 December 2020

Published online: 10 December 2020

\section{References}

1. Gupta RS, Lo B, Son J. Phylogenomics and comparative genomic studies robustly support division of the genus Mycobacterium into an emended genus Mycobacterium and four novel genera. Front Microbiol. 2018;9:67. https://doi.org/10.3389/fmicb.2018.00067.

2. Leão SC, Viana-Niero C, Matsumoto CK, Lima KV, Lopes ML, Palaci M, Hadad DJ, Vinhas S, Duarte RS, Lourenço MC, et al. Epidemic of surgical-site infections by a single clone of rapidly growing mycobacteria in Brazil. Future Microbiol. 2010;5(6):971-80. https://doi.org/10.2217/fmb.10.49.

3. Brown BA, Wallace RJ Jr, Onyi GO, Dr Rosas V, Wallace RJ 3rd. Activities of four macrolides including clarithromycin against Mycobacterium fortuitum, Mycobacterium chelonae, and M. chelonae-like organisms. Antimicrob Agents Chemother. 1992;36(1):180-4. https://doi.org/10.1128/aac.36.1.180.

4. Andrews ER, Marchand-Austin A, Ma J, Cronin K, Sharma MK, Brode SK, Marras TK, Jamieson FB. Underutilization of nontuberculous mycobacterial drug susceptibility testing in Ontario, Canada, 2010-2015. Official J Assoc Med Microbiol Infect Dis Canada. 2020;5(2):77-86. https://doi.org/10.3138/ jammi.2019-0019.

5. Novosad SA, Beekmann SE, Polgreen PM, Mackey K, Winthrop KL. Treatment of Mycobacterium abscessus infection. Emerg Infect Dis. 2016;22(3):511-4. https://doi.org/10.3201/eid2203.150828.

6. Maurer FP, Castelberg C, Quiblier C, Böttger EC, Somoskövi A. Erm(41)dependent inducible resistance to azithromycin and clarithromycin in clinical isolates of Mycobacterium abscessus. J Antimicrob Chemother. 2014; 69(6):1559-63. https://doi.org/10.1093/jac/dku007.

7. Nash KA, Brown-Elliot BA, Wallace RJ Jr. A novel gene, erm(41), confers inducible macrolide resistance to clinical isolates of Mycobacterium abscessus but is absent from Mycobacterium chelonae. Antimicrob Agents Chemother. 2009:53(4):1367-76. https://doi.org/10.1128/AAC.01275-08.

8. Sander P, Prammananan T, Meier A, Frischkorn K, Böttger EC. The role of ribosomal RNAs in macrolide resistance. Mol Microbiol. 1997;26(3):469-80. https://doi.org/10.1046/j.1365-2958.1997.5811946.x.

9. Wallace RJ Jr, Meier A, Brown BA, Zhang Y, Sander P, Onyi GO, Böttger EC. Genetic basis for clarithromycin resistance among isolates of Mycobacterium chelonae and Mycobacterium abscessus. Antimicrob Agents Chemother. 1996;40(7):1676-81.

10. Vester B, Douthwaite S. Macrolide resistance conferred by base substitutions in 23S rRNA. Antimicrob Agents Chemother. 2001;45(1):1-12. https://doi. org/10.1128/AAC.45.1.1-12.2001.

11. Ringuet H, Akoua-Koffi C, Honore S, Varnerot A, Vincent V, Berche P, Gaillard $\mathrm{JL}$, Pierre-Audigier C. hsp65 sequencing for identification of rapidly growing mycobacteria. J Clin Microbiol. 1999;37(3):852-7. https://doi.org/10.1128/ JCM.37.3.852-857.1999.

12. Shallom SJ, Moura NS, Olivier KN, Sampaio EP, Holland SM, Zelazny AM. New real-time PCR assays for detection of inducible and acquired clarithromycin resistance in the Mycobacterium abscessus group. J Clin Microbiol. 2015;53(11):3430-7. https://doi.org/10.1128/JCM.01714-15.

13. Brown-Elliot BA, Vaslreddy S, Vaslreddy R, lakhlaeva E, Howard ST, Nash K, Parodi N, Strong A, Gee M, Smith T, et al. Utility of sequencing the erm(41) gene in isolates of Mycobacterium abscessus subsp. abscessus with low and intermediate clarithromycin MICs. J Clin Microbiol. 2015;53(4):1211-5. https://doi.org/10.1128/JCM.02950-14.
14. Rubio M, March F, Garrigó M, Moreno C, Español M, Coll P. Inducible and acquired clarithromycin resistance in the Mycobacterium abscessus complex. PLoS One. 2015;10(10):e0140166. https://doi.org/10.1371/journal.pone. 0140166.

15. Maurer FP, Rüegger V, Ritter C, Bloemberg GV, Böttger EC. Acquisition of clarithromycin resistance mutations in the 23S rRNA gene of Mycobacterium abscessus in the presence of inducible erm(41). J Antimicrob Chemother. 2012;67(11):2606-11. https://doi.org/10.1093/jac/dks279.

16. Leung KL, Yip CW, Cheung WF, Lo AC, Ko WM, Kam KM. Development of a simple and low-cost real-time PCR method for the identification of commonly encountered mycobacteria in a high throughput laboratory. J Appl Microbiol. 2009;107(5):1433-9. https://doi.org/10.1111/j.1365-2672.2009. 04324.X.

17. Zhu YC, Mitchell KK, Nazarian EJ, Escuyer VE, Musser KA. Rapid prediction of inducible clarithromycin resistance in Mycobacterium abscessus. Mol Cell Probes. 2015;29(6):514-6. https://doi.org/10.1016/j.mcp.2015.08.007.

18. Nakanaga K, Sekizuka T, Fukano H, Sakakibara Y, Takeuchi F, Wada S, Ishii N, Makino M, Kuroda M, Hoshino Y. Discrimination of Mycobacterium abscessus subsp. massiliense from Mycobacterium subsp. abscessus in clinical isolates by multiplex PCR. J Clin Microbiol. 2014;52(1):251-9. https://doi.org/10.1128/ JCM.01327-13.

19. Christianson S, Grierson W, Kein D, Tyler AD, Wolfe J, Sharma MK. Time-todetection of inducible macrolide resistance in Mycobacterium abscessus subspecies and its association with the Erm(41) Sequevar. PLoS One. 2016; 11(8):e0158723. https://doi.org/10.1371/journal.pone.0158723.

20. Kim H, Kim BJ, Kook Y, Yun Y, Shin JH, Kim B, Kook YH. Mycobacterium massiliense is differentiated from Mycobacterium abscessus and Mycobacterium bolletii by erythromycin ribosome methyltransferase gene (erm) and clarithromycin susceptibility patterns. Microbiol Immunol. 2010; 54(6):347-53. https://doi.org/10.1111/j.1348-0421.2010.00221.x.

21. Shallom SJ, Gardina PJ, Myers TG, Sebastian Y, Conville P, Calhoun LB, Tettelin H, Olivier KN, Uzel G, Sampaio EP. New rapid scheme for distinguishing the subspecies of the Mycobacterium abscessus group and identifying Mycobacterium massiliense isolates with inducible clarithromycin resistance. J Clin Microbiol. 2013;51(9):2943-9. https://doi.org/10.1128/JCM. 01132-13.

22. Vianna JS, Machado D, Ramis IB, Silva FP, Bierhals DV, Abril MA, von Groll A, Ramos DF, Lourenço MCS, Viveiros M, da Silva PEA. The contribution of efflux pumps in Mycobacterium abscessus complex resistance to clarithromycin. Antibiotics (Basel). 2019;8(3):153. https://doi.org/10.3390/ antibiotics8030153.

23. Lipworth S, Hough N, Leach L, Morgan M, Jeffery K, Andersson M, Robinson E, Smith EG, Crook D, Peto T, et al. Whole-genome sequencing for predicting clarithromycin resistance in Mycobacterium abscessus. Antimicrob Agents Chemother. 2018;63(1):1204-18. https://doi.org/10.1128/AAC.01204-18.

\section{Publisher's Note}

Springer Nature remains neutral with regard to jurisdictional claims in published maps and institutional affiliations.

Ready to submit your research? Choose BMC and benefit from:

- fast, convenient online submission

- thorough peer review by experienced researchers in your field

- rapid publication on acceptance

- support for research data, including large and complex data types

- gold Open Access which fosters wider collaboration and increased citations

- maximum visibility for your research: over $100 \mathrm{M}$ website views per year

At BMC, research is always in progress.

Learn more biomedcentral.com/submissions 